\title{
POBLACIONES VIABLES Y GRUPOS FUNCIONALES DE HONGOS PRESENTES EN SUELOS DE BOSQUE DE Araucaria-Nothofagus POST-INCENDIO
}

(Viable populations and functional groups of fungi present in soils of AraucariaNothofagus forests, after a fire)

\author{
Oscar Martínez V. ${ }^{\mathbf{1}}$, Eduardo Valenzuela F. ${ }^{\mathbf{1}}$ \& Roberto Godoy B. ${ }^{2}$ \\ ${ }^{1}$ Instituto de Microbiología, ${ }^{2}$ Instituto de Botánica, Facultad de Ciencias \\ Universidad Austral de Chile, Casilla 167, Valdivia.
}

Palabras claves: Hongos, suelo, fuego, Araucaria-Nothofagus, grupos funcionales.

Key words: Fungi, soil, fire, Araucaria-Nothofagus, functional groups.

\section{RESUMEN}

Se determinó después de dos años, el efecto de un incendio natural sobre la población viable y grupos funcionales de hongos del suelo presentes en un bosque de Araucaria asociado a Nothofagus, localizado en el Parque Nacional Tolhuaca. Las poblaciones fúngicas viables fueron aisladas desde el suelo de tres parcelas (PC, P1 y P2) y de dos profundidades (0-20 y 20-40 cm) por el método de las diluciones seriadas y sembrados en agar extracto de malta. La estimación de las poblaciones de los grupos funcionales amonificantes, ureolíticos, amilolíticos y celulolíticos se hizo por el método del número más probable. Se compararon los recuentos y las poblaciones de los grupos funcionales entre las parcelas a través de análisis de varianza (ANOVA). Estadísticamente, no se determinaron diferencias significativas por efecto del fuego sobre los recuentos de las poblaciones fúngicas viables y los grupos funcionales fúngicos presentes. Ambos grupos, no difieren entre áreas quemadas y no quemadas en bosques de Araucaria-Nothofagus. microfungi could not be utilized as potential indicators of the alterations caused by fire on Araucaria forest soil.

\section{INTRODUCCION}

La especie Araucaria araucana (Molina) K. Koch es una conífera endémica de los bosques templados del centro-sur de Chile y Argentina, que forma un bosque de características únicas en el mundo, debido al entorno en
Por lo cual, estos hongos no serían adecuados bioindicadores de la recuperación de este ecosistema.

\begin{abstract}
This study examined the short-term effects of wildfire on fungal communities in an Andean ecosystem dominated by Araucaria araucana in Tolhuaca National Park in southern Chile. An extensive wildfire burned the area in February 2002. Soil samples were collected from two different depths ranges $(0-20$ and 20-40 cm) from a plot not affected by the fire (PC) and from burned plots (P1 and P2). Viable psychrophilic fungi populations were evaluated from these samples. These microorganisms were categorized into four functional groups, ureolytics, ammonifiers, amylolytics, and cellulolytics. The effect of the fire on the soil's microbiological communities was assessed using ANOVA. No statistically significant differences were caused by fire effect on the counts of viable psychrophilic fungi and the functional groups present in Tolhuaca National Park. Therefore, these
\end{abstract}

que se desarrolla, conformado por la cordillera, volcanes andinos nevados y lagos de glaciares (Donoso, 1994).

En Chile, el bosque nativo cubre una superficie de 13.430.602,8 hectáreas, de las cuales 261.073,1 hectáreas (1,9\%) corresponden al bosque de Araucaria (Corporación Nacional Forestal, 2005). La especie A. araucana tiene importancia histórica, taxonómica, ornamental, etno-botánica, ecológica, económica y paleobotánica (Monta-ldo, 1974), por lo cual tiene el estatus de un Monumento Natural. 
La ecología y dinámica de los bosques de $A$. araucana es regulada por perturbaciones naturales, en la Cordillera Andina las principales son: vulcanismo, viento e incendios (Veblen et al., 1996).

Estudios como los de Bissett \& Parkinson (1980) y Mabuhay et al. (2003), han demostrado significativos cambios sobre las comunidades fúngicas y su función en el suelo, como resultado de los incendios forestales, mientras otros estudios han demostrados menores cambios sobre las comunidades fúngicas (Jorgensen \& Hodges, 1970; Tiwari \& Rai, 1977; Deka \& Mishra, 1983). El contraste entre los resultados pueden ser el reflejo de las diferencias entre ecosistemas, y del tiempo y magnitud del incendio estudiado (Staddon et al., 1996). El efecto del fuego sobre las comunidades microbianas del suelo depende de la intensidad (Pietikäinen, 1999) y del tipo de fuego (Barnes et al., 1998). El patrón de comportamiento más frecuente de la microbiota del suelo es una disminución en la cantidad inmediatamente después del incendio, a causa del efecto de esterilización que ejerce el fuego, principalmente en los primeros $5 \mathrm{~cm}$ (Pietikäinen, 1999; Mabuhay et al., 2003). Después del incendio aumenta la concentración de nutrientes y el $\mathrm{pH}$, este último debido a la acumulación de cenizas (Barnes et al., 1998).

Durante el primer mes después del incendio hay una disminución del número de hongos debido al efecto esterilizador del fuego y a la incineración de la materia orgánica (Vázquez et al., 1993). En general, se ha demostrado que los hongos demoran en alcanzar los recuentos poblacionales previos al incendio, debido a la baja cantidad de materia orgánica presente en los suelos afectados por el fuego.

En Chile, el estudio de los microhongos del suelo y su función en los procesos biológicos del suelo, tales como amonificación, degradación de la urea (ureólisis), degradación del almidón (amilólisis) y degradación de la celulosa (celulólisis), después de los incendios en ecosistemas de bosques nativos, no han sido investigados. Dada la vulnerabilidad de estos ecosistemas frágiles y dependientes de estos procesos internos de ciclaje de nutrientes, el conocimiento acerca de las poblaciones fúngicas del suelo y su función, podría proveer de indicadores microbiológicos, para ser usados como potenciales herramientas de monitoreo de la capacidad de recuperación y adaptabilidad de estos bosques frente a eventos catastróficos. En la estación de incendios en la temporada 2001-2002, una importante área de bosques de Araucaria-Nothofagus, fueron severamente afectados por el fuego (Corporación Nacional Forestal, 2002). En particular, el Parque Nacional Tolhuaca fue afectado por un incendio de origen natural que abarco el $60 \%$ de su superficie (González, 2005).
El objetivo del presente estudio fue evaluar el efecto del fuego sobre el número de hongos y grupos funcionales, en suelos de bosque de Araucaria-Notho-fagus, dos años postincendio.

\section{MATERIALES Y METODOS}

\section{Area de estudio}

Corresponde a un bosque de Araucaria-Nothofagus ubicado en el Parque Nacional Tolhuaca $\left(38^{\circ} \mathrm{S}, 72^{\circ} \mathrm{O}\right)$, Chile, que fue afectado por un incendio de origen natural en febrero de 2002 (Corporación Nacional Forestal, 2002). Este Parque esta ubicado en la Provincia de Malleco, IX Región. La superficie total del Parque es de 6.474 hectáreas, cuya altitud es de 700 m.s.n.m. en el límite occidental y 1821 m.s.n.m en el extremo oriental.

De acuerdo a Pollmann (2002), el clima del Parque es templado, frío húmedo, caracterizado por precipitaciones concentradas en invierno y al menos un mes en verano sin lluvias. La precipitación media anual es de aproximadamente $2.500 \mathrm{~mm}$, incluyendo nieve, entre los meses de junio y septiembre. La temperatura media mensual tiene un rango de $19,9^{\circ} \mathrm{C}$ en enero a $7,0^{\circ} \mathrm{C}$ en julio, con un promedio anual de $12^{\circ} \mathrm{C}$. Desde el punto de vista geológico, el área del Parque pertenece al complejo volcánico formado por los volcanes Tolhuaca y Lonquimay (Pollmann, 2002). Los suelos se formaron a partir de sedimentos volcánicos recientes. Estos suelos se clasifican como andisoles del subtipo Vitrands y en el grupo de (Thaphic-)Udivitrands (Pollmann, 2001).

\section{Parcelas experimentales y colecta del suelo}

Para estudiar el impacto del fuego sobre el ecosistema boscoso Araucaria-Nothofagus, se contrastó los resultados de las variables medidas entre los sitios quemados con el no quemado, que corresponde al control. Para lo cual se establecieron en el lugar 3 parcelas, un control denominado (PC) y dos afectadas por el fuego (P1 y P2). Se hicieron muestreos estacionales, réplicas temporales de las parcelas, en verano, otoño, invierno y primavera. De cada parcela se extrajeron 3 muestras de suelo, de $500 \mathrm{~g}$, desde las profundidades $0-20 \mathrm{~cm}$ y 20-40 $\mathrm{cm}$. Las muestras de suelo fueron depositadas independientemente en bolsas plásticas y mantenidas en un contenedor refrigerante, para su traslado. Posteriormente las muestras fueron tamizadas a $2 \mathrm{~mm}$, homogenizadas y acondicionadas. Se conformó una muestra compuesta para cada caso, mezclando las 3 submuestras de cada profundidad y respectiva parcela.

Los análisis microbiológicos se realizaron dentro de las primeras 48 horas desde la colecta de suelo.

\section{Recuento de hongos.}


Cada muestra compuesta de suelo fue procesada en forma independiente, para ello se pesaron $10 \mathrm{~g}$ de la muestra y se depositaron en un matraz que contenía 100 $\mathrm{mL}$ de agua destilada estéril, la mezcla obtenida se agitó vigorosamente y a partir de ella se realizaron diluciones seriadas hasta la $10^{-7}$, de acuerdo al método que aparece descrito por Parkinson et al. (1971) y Parkinson (1994). Para el recuento de los hongos, se depositó en una placa Petri $1 \mathrm{~mL}$ de la dilución respectiva, y se le adicionó 0,2 $\mathrm{mL}$ de una mezcla de antibióticos $(50 \mu \mathrm{g} / \mathrm{mL}$ de Penicilina y $25 \mu \mathrm{g} / \mathrm{mL}$ de Estreptomicina) y $10 \mathrm{~mL}$ de agar extracto de malta al $2 \%$ (AEM) enfriado a $45{ }^{\circ} \mathrm{C}$, las placas sembradas se incubaron a $23 \pm 2{ }^{\circ} \mathrm{C}$ por 7 días, tiempo al que se efectuaron los recuentos. El recuento expresa el número de propágulos fúngicos (PF) por gramo de suelo seco (PF gss $\left.{ }^{-1}\right)$. Cada dilución fue sembrada por triplicado.

\section{Evaluación de grupos funcionales fúngicos.}

Se realizaron de acuerdo al método del Número Más Probable (NMP) que aparece descrito por Pochon y Tardieux (1965) y Woomer (1994). Las poblaciones fúngicas para cada grupo funcional se determinaron por el método del NMP por g de suelo seco (NMP gss-1), para lo cual se consultó la tabla de McCrady para tres repeticiones (3 tubos) por dilución sembrada. Se procedió a contar, para cada dilución, el número de tubos positivos. De este modo se obtuvo un número característico de tres dígitos, el que se buscó en la tabla de MacCrady el NMP de gérmenes.

Población fúngica amonificante. Se realizó de acuerdo al método señalado por Pochon y Tardieux (1965). De la respectiva dilución de suelo se tomó $1 \mathrm{~mL}$ y se depositó en un tubo que contenía $5 \mathrm{~mL}$ de caldo asparragina más 0,2 mL de la mezcla de antibióticos (50 $\mu \mathrm{g} / \mathrm{mL}$ de Penicilina y $25 \mu \mathrm{g} / \mathrm{mL}$ de Estreptomicina). Cada dilución se sembró por triplicado. Luego de la siembra los tubos se incubaron a $23 \pm 2{ }^{\circ} \mathrm{C}$, por 20 días. Al cabo de este período a los tubos se le agregaron $0,2 \mathrm{~mL}$ del reactivo de Nessler. Una reacción positiva da una coloración amarillo-naranja.

Población fúngica ureolítica. Se realizó de acuerdo al método señalado por Pochon y Tardieux (1965) y MacFaddin (1976). De la respectiva dilución de suelo se tomó $1 \mathrm{~mL}$ y se depositó en un tubo que contenía $4 \mathrm{~mL}$ de caldo urea más $0,2 \mathrm{~mL}$ de la mezcla de antibióticos (50 $\mu \mathrm{g} / \mathrm{mL}$ de Penicilina y $25 \mu \mathrm{g} / \mathrm{mL}$ de Estreptomicina). Cada dilución se sembró por triplicado. Luego de la siembra los tubos se incubaron a $23 \pm 2{ }^{\circ} \mathrm{C}$, por 14 días. Al cabo de este período los tubos positivos dieron una coloración rosada.

Población fúngica amilolítica. Se realizó de acuerdo al método señalado por Pochon \& Tardieux (1965). De la respectiva dilución de suelo se tomó $1 \mathrm{~mL}$ y se depositó en un tubo que contenía $4 \mathrm{~mL}$ caldo de almidón más $0,2 \mathrm{~mL}$ de la mezcla de antibióticos $(50 \mu \mathrm{g} / \mathrm{mL}$ de Penicilina y $25 \mu \mathrm{g} / \mathrm{mL}$ de Estreptomicina). Cada dilución se sembró por triplicado. Luego de la siembra los tubos se incubaron a $23 \pm 2{ }^{\circ} \mathrm{C}$, por 15 días. Al cabo de este período a los tubos se le agregaron $0,4 \mathrm{~mL}$ del reactivo yodo-yodurado (Lugol). Una reacción positiva mostró una coloración con un ligero tinte amarillo-naranja.

Población fúngica celulolítica. Se realizó de acuerdo al método señalado por Pochon \& Tardieux (1965). De las respectivas diluciones de suelo, se tomó 1 $\mathrm{mL}$ y se depositó en una placa que contenía agar celulosa. Se sembraron 3 placas por dilución. Una vez sembradas las placas se incubaron a $23 \pm 2{ }^{\circ} \mathrm{C}$ por 14 días. Las positivas tuvieron crecimiento visible de colonias de hongos.

\section{Análisis estadístico}

Los datos obtenidos sobre el recuento de hongos y grupos funcionales fúngicos, fueron sometidos a análisis de varianza de una vía (ANOVA) para determinar diferencias entre parcelas. Cuando se comparó las dos profundidades de muestreo del suelo, se utilizó la prueba t de Student. Además, se aplicó el test de Levene para verificar homogeneidad de varianza de los datos, y cuando fue necesario se hicieron transformaciones a los datos.

\section{RESULTADOS}

\section{Población fúngica}

El recuento de las poblaciones fúngicas después de dos años de ocurrido el incendio en el bosque de Araucaria-Nothofagus, Parque Nacional Tolhuaca se observan en la Figura 1. En la profundidad 0-20 cm, el mayor recuento $\left(1,3 \times 10^{6} \mathrm{PF}\right.$ gss $\left.^{-1}=\log _{10} 6,11\right)$ de propágulos fúngicos se registró en la parcela $\mathrm{PC}$ y el menor $\left(5,6 \times 10^{4} \mathrm{PF} \mathrm{gss}^{-1}=\log _{10} 4,75\right)$ en P2. En la profundidad

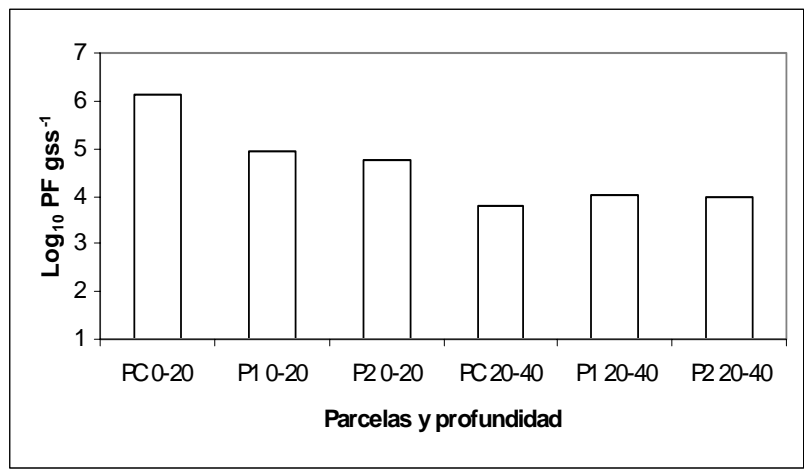

Figura 1. Recuento promedio de propágulos fúngicos $\left(\log _{10}\right.$ PF gss $\left.{ }^{-1}\right)$ en el bosque de AraucariaNothofagus, Parque Nacional Tolhuaca, durante el año 2004. 
20-40 cm, el mayor recuento $\left(1,0 \times 10^{4} \mathrm{PF} \mathrm{gss}^{-1}=\log _{10} 4,02\right)$ se registró en $\mathrm{P} 1$, y el menor $\left(5,9 \times 10^{3} \mathrm{PF} \mathrm{gss}^{-1}=\log _{10}\right.$ 3,78) en la parcela PC.

El análisis ANOVA (datos transformados a $\log _{10}$ ) no mostró diferencias estadísticas significativas $(F=1,369$, $p=0,303$ ) en el recuento de propágulos fúngicos entre las parcelas en los primeros $20 \mathrm{~cm}$, para el período en estudio. Para la profundidad 20-40 cm, el análisis ANOVA no mostró diferencias significativas $(F=0,22, p=0,807)$. Al comparar el recuento de propágulos fúngicos determinados a ambas profundidades, los resultados de la prueba $t$ (datos transformados a $\log _{10}$ ), muestran que hay diferencias estadísticas significativas ( $p=0,006)$. Registrándose los mayores recuentos en las muestras de suelo de la profundidad $0-20 \mathrm{~cm}$.

\section{Grupos funcionales}

Hongos amonificantes: Las poblaciones fúngicas amonificantes, presentes dos años post incendio en el bosque de Araucaria-Nothofagus se observan en la Figura 2A. En la profundidad 0-20 cm, la mayor población (4,3 $\left.\mathrm{x} 10^{5} \mathrm{NMP} \mathrm{gss}^{-1}=\log _{10} 5,63\right)$ se registró en PC, y la menor $\left(3 \times 10^{5} \mathrm{NMP} \mathrm{gss}^{-1}=\log _{10} 5,48\right)$ en P2. En la profundidad 20-40 cm (Figura 2A), la mayor población (3,6 x10 $\mathrm{NMP}$ $\left.\mathrm{gss}^{-1}=\log _{10} 5,57\right)$ se registró en P1, y la menor $\left(1,3 \times 10^{5}\right.$

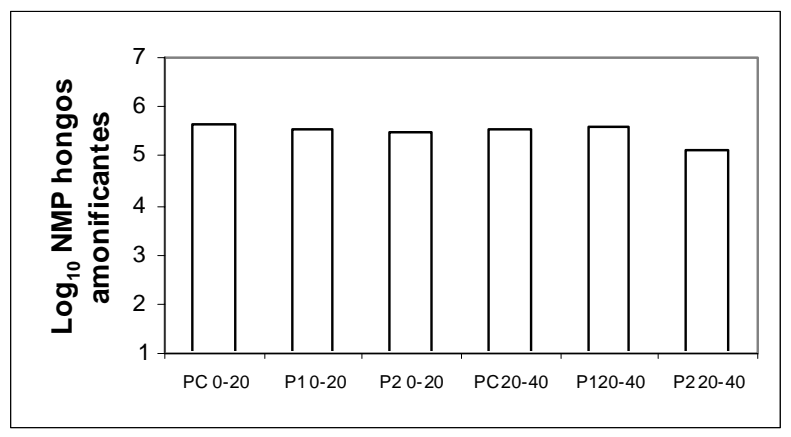

A

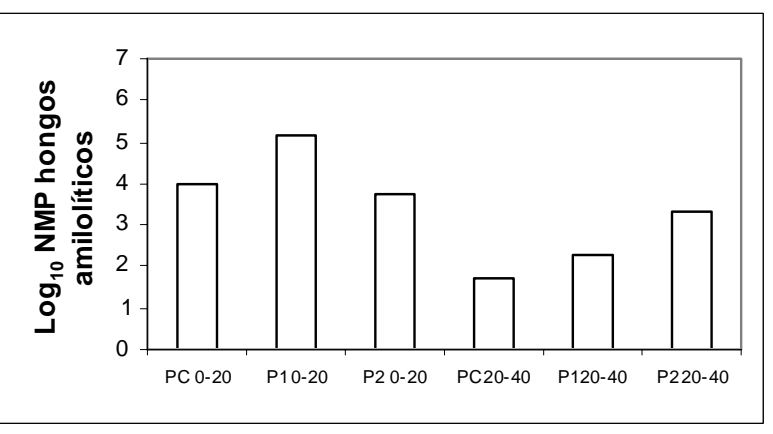

NMP gss $\left.{ }^{-1}=\log _{10} 5,14\right)$ en P2. El análisis ANOVA no determinó diferencias estadísticas entre las parcelas a profundidades de $0-20 \mathrm{~cm}(F=0,678, p=0,532)$ ni a 20 $40 \mathrm{~cm}(F=1,24, p=0,334)$.

$\mathrm{Al}$ comparar las poblaciones presentes en las dos profundidades (0-20 y 20-40 cm), los resultados de la prueba $t$ mostraron que no hay diferencia estadística ( $p$ $=0,416$ ).

Hongos ureolíticos: En la profundidad $0-20 \mathrm{~cm}$, la mayor población $\left(8,8 \times 10^{4} \mathrm{NMP} \mathrm{gss}^{-1}=\log _{10} 4,95\right)$ se registró en P2 y la menor en PC $\left(5,7 \times 10^{4} \mathrm{NMP} \mathrm{gss}^{-1}=\right.$ $\left.\log _{10} 4,76\right)$. A $20-40 \mathrm{~cm}$, la mayor población $\left(11,9 \times 10^{4}\right.$ NMP gss $\left.{ }^{-1}=\log _{10} 5,08\right)$ se registró en P1, y la menor $(1,2$ $\mathrm{x} 10^{3} \mathrm{NMP} \mathrm{gss}^{-1}=\log _{10} 3,09$ ) en PC (Figura 2B). El análisis ANOVA no determinó diferencias estadísticas en la profundidad $0-20 \mathrm{~cm}(F=0,206, p=0,818)$ ni en la profundidad 20-40 $\mathrm{cm}(F=1,407, p=0,294)$. Al comparar las poblaciones de las dos profundidades (0-20 y 20-40 $\mathrm{cm})$, los resultados de la prueba t mostraron que no hay diferencia estadística $(p=0,646)$.

Hongos amilolíticos: En la profundidad 0-20 cm, la mayor población $\left(1,4 \times 10^{5} \mathrm{NMP}\right.$ gss $\left.^{-1}=\log _{10} 5,17\right)$ se registró en P1, y la menor $\left(5,6 \times 10^{3} \mathrm{NMP}\right.$ gss $^{-1}=\log _{10}$

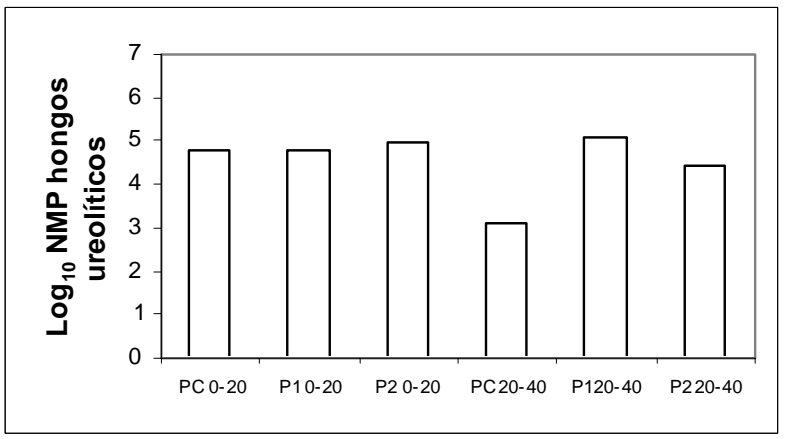

B

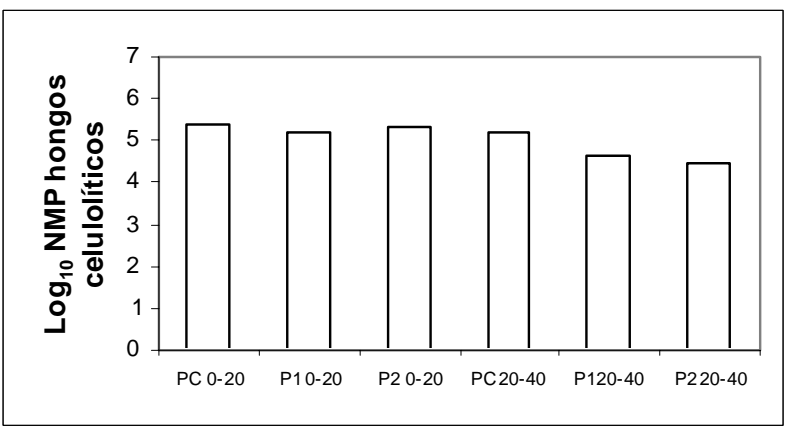

Figura 2. Recuentos fúngicos $\left(\log _{10} \mathrm{NMP}\right.$ gss $\left.^{-1}\right)$ promedios: A) población amonificante, B) población ureolítica, C) población amilolítica y D) población celulolítica. 
3,75) en P2. En la profundidad 20-40 cm, la mayor población $\left(1,9 \times 10^{3} \mathrm{NMP}\right.$ gss $\left.^{-1}=\log _{10} 3,3\right)$ se registró en P2, y la menor (55 NMP gss ${ }^{-1}=\log _{10} 1,74$ ) en PC (Figura 2 C). El análisis ANOVA (datos transformados a $\log _{10}(\mathrm{NMP})$ ) no determinó diferencias estadísticas entre las poblaciones amilolíticas de las parcelas a ninguna de las dos profundidades $(F=0,037, p=0,964$ en 0 -20 cm y $F=1,595, p$ $=0,225$ en $20-40 \mathrm{~cm}$ ). Al comparar las poblaciones de las dos profundidades, los resultados de la prueba $\mathrm{t}$ (datos transformados a $\left.\log _{10}(\mathrm{NMP}+1)\right)$ mostraron que hay diferencia estadística ( $p=0,002)$, siendo mayor en 0-20 cm.

Hongos celulolíticos: En la profundidad 0-20 cm, la mayor población $\left(2,4 \times 10^{5} \mathrm{NMP}\right.$ gss $\left.^{-1}=\log _{10} 5,39\right)$ se registró en PC, y la menor $\left(1,6 \times 10^{5} \mathrm{NMP}\right.$ gss $^{-1}=\log _{10}$ $5,21)$ en P1. A $20-40 \mathrm{~cm}$, la mayor población $\left(1,7 \times 10^{5}\right.$ NMP gss $\left.{ }^{-1}=\log _{10} 5,23\right)$ se registró en PC, y la menor $(2,9$ $\mathrm{x} 10^{4} \mathrm{NMP}$ gss $^{-1}=\log _{10} 4,47$ ) en P2 (Figura 2D). El análisis ANOVA no determinó diferencias estadísticas entre estas poblaciones a ninguna de las dos profundidades $(F=0,165, p=0,850$ en $0-20 \mathrm{~cm}$ y $F=2,677$, $p=0,122$ en $20-40 \mathrm{~cm})$. Al comparar las poblaciones presentes en las dos profundidades (0-20 y 20-40 cm), los resultados de la prueba $t$ (datos transformados a $\log _{10}(\mathrm{NMP})$ ) mostraron que hay diferencia estadística ( $p$ $=0,041$ ), siendo mayor la población en los primeros 20 $\mathrm{cm}$.

\section{DISCUSION}

\section{Recuento de hongos}

Entre las parcelas no hubo diferencia estadísticamente significativa respecto al recuento de las poblaciones fúngicas del suelo en el bosque de AraucariaNothofagus estudiado, después de dos años transcurridos desde el incendio. Esto concuerda con Vásquez et al. (1993), quienes señalan que un mes después del incendio el número de hongos presentes en el suelo quemado disminuyeron 19 veces respecto al suelo control, sin embargo, transcurrido un año después del incendio los hongos presentes en el suelo quemado aumentaron, y solo disminuyeron 2 veces en relación al suelo control. Por otro lado, Jorgensen \& Hodges (1970), indican que el fuego no alteró a las poblaciones fúngicas en suelos de bosque de Pinus taeda. Por su parte, Deka \& Mishra (1983) observaron que los hongos alcanzaron los niveles poblaciones iniciales un mes después del incendio, mientras que Tiwari \& Rai (1977), indican una recuperación de la población fúngica a los dos meses postincendio. Al contrario, Mabuhay et al. (2003), estudiaron un bosques de pino, donde compararon un área no quemada (control) y dos áreas quemadas, una inmediatamente después del incendio y otra dos años después del incendio. El mayor recuento fúngico fue determinado en el área control, seguido del área donde el incendio ocurrió hace dos años, y el menor recuento de propágalos fúngicos fue en el área en que el incendio fue más reciente. Según Vázquez et al. (1993), los factores que tendrían influencia en la recuperación de las poblaciones fúngicas iniciales son el aumento en el pH y la destrucción de la materia orgánica, producto del incendio.

En el presente estudio los resultados del recuento de hongos muestran diferencias estadísticas significativas de acuerdo a la profundidad de la muestra de suelo analizada, se percibe una disminución de los propágalos fúngicos con el aumento de la profundidad. Esto como lo han señalado Alexander (1980), Deka \& Mishra (1983) y Paul \& Clark (1996), probablemente se debe a una disminución del contenido de materia orgánica y oxígeno.

Grupos funcionales fúngicos. De acuerdo con Campbell (1987) y Andrade (2004), los grupos funcionales de microorganismos estudiados en la presente investigación pertenecen a los ciclos del nitrógeno y del carbono. Estos grupos funcionales fúngicos se encuentran en un estado dinámico de equilibrio en bosques maduros. Sin embargo, Acea \& Carballas (1996) y Fisher \& Binkley (2000), indican que este equilibrio es alterado por los incendios forestales, los que provocan la destrucción de los sustratos orgánicos, afectando en el corto plazo la composición de la comunidad microbiana del suelo y su función. La recuperación del rol funcional de las comunidades microbianas en los ecosistemas boscosos, esta asociado a la regeneración de la nueva vegetación y la acumulación de litera y hojarasca (Paul \& Clark, 1996; Hart et al., 2005).

En el ecosistema Araucaria-Nothofagus, que se caracteriza por ser limitado en N (Lusk, 2001), la actividad amonificante (conversión del nitrógeno orgánico en amonio o amoniaco) y ureolítica de los microorganismos del suelo está involucrada en el aprovechamiento eficiente de formas orgánicas de nitrógeno. La mineralización del nitrógeno orgánico es esencial para que el ecosistema siga siendo productivo (Atlas \& Bartha, 2002). Respecto al efecto del fuego sobre las poblaciones amonificantes en bosque de Pinus pinaster, Acea \& Carballas (1996), observaron que un mes después del incendio los microorganismos amonificantes aumentaron 32 veces respecto al sitio control, al año después del fuego la población amonificante disminuyó 16 veces respecto al tamaño de la población un mes después del incendio. La alta población amonificante encontrada en bosques de Araucaria-Nothofagus, probablemente debido a la redundancia funcional, concuerda con Acea \& Carballas (1990), quienes indican que el 99\% de los microor- 
ganismos presentes en suelos de distintos bosques tienen la capacidad de realizar amonificación.

Mobley \& Hausinger (1989), señalan que la actividad ureolítica microbiana esta ampliamente distribuida en el suelo e incluye la acción de bacterias y hongos. Esta actividad decrece por efecto del fuego, como lo observaron Hernández et al. (1997), en bosques de pino, en el mediterráneo.

Respecto al efecto del fuego sobre la población amilolítica en bosque de Pinus pinaster, Acea \& Carballas (1996), señalan que en el corto plazo el fuego favorece a los microorganismos descomponedores de almidón. Un mes después del incendio la población amilolítica presente en el suelo quemado era 2,5 veces mayor que el control, al año la población amilolítica era la mitad de lo que se registró el primer mes después del fuego, e inferior al control. La capacidad de degradar almidón es usualmente común entre las poblaciones microbianas del suelo (Wagner \& Wolf, 1998; Andrade, 2004). El incremento de la población amilolítica en las parcelas afectadas por el fuego, probablemente se debe al aumento de las poblaciones fúngicas y bacterias después del incendio (Vázquez et al. ,1993).

Respecto al efecto del fuego sobre la población celulolítica en bosque de Pinus pinaster, Acea \& Carballas (1996), indican que un mes después del incendio, el número de microorganismos celulolíticos disminuyó 2.515 veces (3 ordenes de magnitud logarítmica), en relación al control. Un año después del incendio, la población celulolítica permanecía baja en relación al control, pero aumentó en 8 veces en relación al tamaño de la población, que se registró el primer mes después del incendio. La recuperación de las poblaciones celulolíticas después del fuego esta asociada a la incorporación de residuos vegetales al suelo.

\section{CONCLUSIONES}

En conclusión y considerando que este trabajo se realizó después de 2 años de ocurrido el incendio, la respuesta de las poblaciones fúngicas viables y los grupos funcionales (posiblemente psicrófilos o psicotolerantes) obtenidos de suelos con bajas temperaturas en bosques de Araucaria-Nothofagus, no difieren entre áreas quemadas y no quemadas. De acuerdo a estos resultados, este tipo de hongos no serían adecuados bioindicadores de la recuperación de este ecosistema.

\section{AGRADECIMIENTOS}

Al Proyecto Bilateral de Cooperación Científica y Tecnológica entre la Univ. Austral de Chile y la Univ. de Gent, Comunidad Flamenca de Bélgica, cuyo título es: «Effect of Fire Damage on Regeneration and N Loss from
Araucaria araucana (Mol.) K. Koch Forest in Southern Chile». A la Corporación Nacional Forestal (CONAF) de la IX Región y a la Red Iberoamericana sobre diversidad, ecología y uso de los hongos microscópicos (REDEMIC).

\section{REFERENCIAS}

Acea, M. \& Carballas, T. (1990). Principal components analysis of the soil microbial population of humic zone of Galicia (Spain). Soil Biol. Biochem. 22:749-759

Acea, M. \& Carballas, T. (1996). Changes in physiological groups of microorganisms in soil following wildfire. FEMS Microbiol. Ecol. 20:33-39

Alexander, M. (1980). Microbiología de los suelos. SKF Editor S.A. Madrid, España.

Andrade, G. (2004). Role of functional groups of microorganisms on the rhizosphere microcosm dynamics. En: A. Varma, L. Abbott, D. Werner \& R. Hampp (eds.). Plant surface microbiology. Springer. pp. 51-69

Atlas, R. \& Bartha, R. (2002). Ecología microbiana y microbiología ambiental. $4^{\text {a }}$ edición. Addison Wesley.

Barnes, B.; Zak, D.; Denton, S. \& Spurr, S. (1998). Forest Ecology. Fourth Edition. John Wiley \& Sons.

Bissett, J. \& Parkinson, D. (1980). Long-term effects of fire on the composition and activity of the soil microflora of a subalpine, coniferous forest. Can. J. Bot. 58:1704-1721

Campbell, R. (1987). Ecología microbiana. Primera edición. Editorial Limusa S.A. Mexico.

Corporación Nacional Forestal. (2002). Antecedentes sobre impacto de incendios forestales en la IX región. Documento de la Visita a Zona Afectada por Incendios, Cap. IV: Principales áreas silvestres protegidas y zonas aledañas afectadas en temporada 20012002, IX Región. CONAF Temuco, Chile. pp 1-13.

Corporación Nacional Forestal. (2005). Superficie Nacional de Bosque Nativo, según Tipo Forestal. http://www.conaf.cl. Revisado el 20 de diciembre de 2005.

Deka, H. \& Mishra, R. (1983). The effects of slash burning on soil microflora. Plant and Soil 73:167-175

Donoso, C. (1994). Bosques templados de Chile y Argentina. Segunda edición. Editorial Universitaria.

Fisher, R. \& Binkley, D. (2000). Ecology and Management of Forest Soils, 3th Edition. John Wiley \& Sons.

González, M. (2005). Fire history data as reference information in ecological restoration. Dendrochronologia 22:149-154

Hart, S.; DeLuca,T.; Newman, G.; MacKenzie, M.; Boyle, S. (2005). Post-fire vegetative dynamics as drivers of microbial community structure and function in forest soils. Forest Ecol. Manag. 220:166-184

Hernández, T.; García, C. \& Reinhardt, I. (1997). Short-term effect of wildfire on the chemical, biochemical and microbiological properties of Mediterranean pine forest soils. Biol. Fertil. Soils 25:109-116 
Jorgensen, J. \& Hodges, C. (1970). Microbial characteristics of a forest soil after twenty years of prescribed burning. Mycologia $62: 721-726$

Lusk, C. (2001). Leaf life spans some conifers of the temperate forest of South America. Rev. Chil. Hist. Nat. 74:711-718

Mabuhay, J.; Nakagoshi, N. \& Horokoshi, T. (2003). Microbial biomass and abundance after forest fire in pine forests in Japan. Ecol. Res. 18:431-441

MacFaddin, J. (1976). Biochemical tests for identification of medical bacteria. The Williams \& Wilkins Company, Baltimore.

Mobley, H. \& Hausinger, R. (1989). Microbial Ureases: Significance, Regulation, and Molecular Characterization. Microbiol. Rev. 53:85108

Montaldo, P. (1974). La bio-ecología de Araucaria Araucana (Mol) Koch. Instituto Forestal Latino-Americano 46/48:3-55

Parkinson, D.; Gray, T.; Holding, J. \& Nagel-de-Boois, H. (1971). Heterotrophic Microflora. En: J. Phillipson (ed.). Methods of Study in Quantitative Soil Ecology: population, production and energy flow. Blackwell Scientific Publications.

Parkinson, D. (1994). Filamentous fungi. En: R. Weaver, S. Angle, P. Bottomley, D. Bezdicek; S. Smith, A. Tabatabai and A. Wollum (eds). Methods of Soil Analysis. Part 2, Microbiological and Biochemical Properties. Number 5 in Soil Science Society of America Book Series. Soil Science Society of America, Inc., Madison, Wisconsin, USA. pp. 329-350

Paul, E. \& Clark, F. (1996). Soil microbiology and biochemistry. Second edition. Academic Press.

Pietikäinen, J. (1999). Soil microbes in boreal forest humus after fire. Academic Dissertation in Forest Soil Science. Finnish Forest Research Institute. Faculty of Agriculture and Forestry, University of Helsinki.
Pochon, J. \& Tardieux, P. (1965). Técnicas de análisis en microbiología del suelo. Editorial T.E.I. Burgos.

Pollmann, W. (2001). Caracterización florística y posición sintaxonómica de los bosques caducifolios de Nothofagus alpina (Poepp.et Endl.) en el centro-sur de Chile. Phytocoenologia 31:353400

Pollmann, W. (2002). Effects of natural disturbance and selective logging on Nothofagus forest in south-central Chile. J. Biogeography 29:955-970

Staddon, W.; Duchesne, L. \& Trevors, J. (1996). Conservation of forest soil microbial diversity: the impact of fire and research needs. Environ. Rev. 4:267-275

Tiwari, V. \& Rai, B. (1977). Effects of soil burning on microfungi. Plant and Soil 47:693-697

Vázquez, F.; Acea, M. \& Carballas, T. (1993). Soil microbial populations after wildfire. FEMS Microbiol. Ecol. 13:93-104

Veblen, T.; Kitzberger,T.; Burns, B. \& Rebertus, A. (1996). Perturbaciones y dinámica de regeneración en bosques andinos del sur de Chile y Argentina. En: J. Armesto, C. Villagrán y M. Arroyo (eds.). Ecología de los bosques nativos de Chile. Editorial Universitaria. pp 169-198

Wagner, G. \& Wolf, D. (1998). Carbon transformations and soil organic matter formation. In: D.M. Sylvia, J.J. Furhmann, P.G. Hartel and D.A. Zuberer (eds.). Principles and applications of soil microbiology. Prentice Hall, Englewood Cliffs. pp. 218-258

Woomer, P. (1994). Most probable number counts. En: R. Weaver, S. Angle, P. Bottomley, D. Bezdicek; S. Smith, A. Tabatabai and A. Wollum (eds). Methods of Soil Analysis. Part 2, Microbiological and Biochemical Properties. Number 5 in Soil Science Society of America Book Series. Soil Science Society of America, Inc., Madison, Wisconsin, USA. pp. 59-80 